\title{
Transformer fault diagnosis based on chemical reaction optimization algorithm and relevance vector machine
}

\author{
Wei Luo ${ }^{1}$, Minfang Peng ${ }^{1}$, Xun Wan ${ }^{2}$, Shengquan Wang ${ }^{3}$ and Xiupu Liao ${ }^{1}$ \\ ${ }^{1}$ College of Electrical and Information Engineering, Hunan University, Changsha 410082, Hunan Province, China \\ ${ }^{2}$ State Grid Hunan Electric Power Corporation Research Institute, Changsha 410007, Hunan Province, China \\ ${ }^{3}$ State Grid Jiangsu Electric Power Corporation Research Institute, Nanjing 211103, Jiangsu Province, China
}

\begin{abstract}
Power transformer is one of the most important equipment in power system. In order to predict the potential fault of power transformer and identify the fault types correctly, we proposed a transformer fault intelligent diagnosis model based on chemical reaction optimization (CRO) algorithm and relevance vector machine(RVM). RVM is a powerful machine learning method, which can solve nonlinear, high-dimensional classification problems with a limited number of samples. CRO algorithm has well global optimization and simple calculation, so it is suitable to solve parameter optimization problems. In this paper, firstly, a multi-layer RVM classification model was built by binary tree recognition strategy. Secondly, CRO algorithm was adopted to optimize the kernel function parameters which could enhance the performance of RVM classifiers. Compared with IEC three-ratio method and the RVM model, the CRO-RVM model not only overcomes the coding defect problem of IEC three-ratio method, but also has higher classification accuracy than the RVM model. Finally, the new method was applied to analyze a transformer fault case, Its predicted result accord well with the real situation. The research provides a practical method for transformer fault intelligent diagnosis and prediction.
\end{abstract}

\section{Introduction}

The power transformer is the core equipment in power system, so it is very important to analyze its operating parameters and make a correct judgment on the internal latent faults. The research on intelligent fault diagnosis of transformer is the key of ensuring power system safety [1].

The operating environment of power transformers are complicated, which has subjected by heat and electricity. Transformer insulation oil will be decomposed to corresponding gases by the process of heat and electricity. The gas types and their volume fraction can distinguish the fault types effectively. Now, dissolved gas analysis (DGA) is a convenient way to analyze and diagnose the potential fault of transformer [2,3]. The classical IEC three-ratio method is widely used because of its simple principle. However, its coding mode is too absolute, which causes missed diagnosis. Therefore, how to improve diagnosis accuracy becomes a research focus in the field of transformer fault diagnosis [4].

In recent years, with the rapid development of artificial intelligence, many scholars are committed to the research of the transformer fault diagnosis based on artificial intelligence algorithm combined with DGA. For example, artificial neural network(ANN) was widely used in Transformer fault diagnosis [5]. However, it requires a lot of training samples and its convergence rate is too slow. Also, support vector machine(SVM) has advantages in dealing with nonlinear and small sample data classification problems[6, 7]. Thus it has made some achievements in transformer fault diagnosis. But its deficiency is that the number of support vectors will increase sharply with the sample size expanding, and the kernel function parameters are restricted by Mercer condition [8].

Relevance Vector Machine (RVM) is a new supervised learning method, which is proposed by Michael E. Tippping [9]. RVM is a sparse learning algorithm based on Bayesian framework. Compared to SVM, the number of relevance vectors is greatly reduced, and the kernel function is no longer constrained by the Mercer condition. At present, RVM has been applied to signal processing and power load forecasting, but it is rarely used in transformer fault diagnosis. This paper presents a multi-layer classifier model based on relevance vector machine, then combined with chemical reaction optimization algorithm to optimize the parameter of kernel functions in each layer. Finally, this paper collected 300 sets of measured data, then we use IEC three ratio method, the standard RVM model and the CRO-RVM model to test these data sets. The results show that the diagnostic model presented in this paper has obvious advantages over other models in diagnostic accuracy. And through some diagnosis cases, we found it is suitable for practical application. 


\section{Introduction of relevance vector machine multi-classification model}

\subsection{Relevance vector machine (RVM)}

Hyper parameter is introduced into RVM machine learning algorithm to ensure its sparsity. It can assign zero-mean Gaussian prior distribution to the weight vector. The maximizing marginal likelihood function method is applied to estimate hyper parameter, and the automatic regulation mechanism is adopted to avoid the problem of the difficulty in determining the regularization coefficient $[10,11]$.

Let $\mathbf{t}=\left[t_{1}, t_{2}, \cdots t_{N}\right]^{T}$ be objective function and $\left\{x_{i}\right\}_{i=1}^{N}$ be input vector, then the RVM classification model can be expressed as the following equation (1):

$$
y(\mathbf{x}, \mathbf{w})=\sum_{i=1}^{N} w_{i} K\left(\mathbf{x}, \mathbf{x}_{i}\right)+w_{0}
$$

where $\mathbf{w}$ is weight vector, $\mathbf{w}=\left[w_{0}, w_{1}, \cdots, w_{N}\right]^{T} ;$ and $K\left(\mathbf{x}, \mathbf{x}_{i}\right)$ is kernel function .

For a two classification problem, the target value is only 0 or 1 , so we can use formula (2) to characterize the likelihood function of the whole data sets:

$$
P(\mathbf{t} \mid \mathbf{w})=\prod_{i=1}^{N} \sigma\left[y\left(\mathbf{x}_{i}, \mathbf{w}\right)\right]^{t_{i}}\left\{1-\sigma\left[y\left(\mathbf{x}_{i}, \mathbf{w}\right)\right]\right\}^{1-t_{i}}
$$

Where $\sigma(\bullet)$ is a sigmoid function.

Sparse Bayesian method is used to give zero-mean Gaussian prior distribution for weight vector w, which can avoid over-fitting and keep the model sparse:

$$
p(\mathbf{w} \mid \boldsymbol{\alpha})=\prod_{i=0}^{N} N\left(w_{i} \mid 0, \alpha_{i}^{-1}\right)
$$

where $\alpha$ is a $\mathrm{N}+1$ dimensional hyper parameter vector, $\boldsymbol{\alpha}=\left(\alpha_{0}, \alpha_{1}, \cdots, \alpha_{N}\right)^{T}$; and $N(\bullet)$ represents the normal distribution function.

For a new input vector $\mathbf{x}$, the probability prediction expression of objective value $t$ is given by the following equation (4) :

$$
p\left(\mathbf{t}_{*} \mid \mathbf{t}\right)=\int p\left(\mathbf{t}_{*} \mid \mathbf{w}, \boldsymbol{\alpha}\right) p(\mathbf{w} \mid \mathbf{t}, \boldsymbol{\alpha}) p(\alpha \mid t) d w d \alpha
$$

Since the weighted posterior probability $p(\mathbf{w} \mid \mathbf{t}, \boldsymbol{\alpha})$ and the marginal likelihood function $p(\boldsymbol{\alpha} \mid \mathbf{t})$ in formula (4) can not be solved by integration, the approximate solution proposed by Mackay needs to be used. The specific steps of this method are as follows.

1)Firstly, assign a value to the current hyper parameter $\boldsymbol{\alpha}$, then the "most likely" weight $\mathbf{w}_{M P}$ is estimated by using the maximum weight posterior probability function $p(\mathbf{w} \mid \mathbf{t}, \boldsymbol{\alpha})$. since $p(\mathbf{w} \mid \mathbf{t}, \boldsymbol{\alpha}) \propto p(\mathbf{t} \mid \mathbf{w}) p(\mathbf{w} \mid \boldsymbol{\alpha})$, the following equation (5) can be used to estimated the weight value of $\mathbf{w}_{M P}$.

$$
\begin{aligned}
& \log [p(\mathbf{t} \mid \mathbf{w}) p(\mathbf{w} \mid \boldsymbol{\alpha})= \\
& \sum_{i=1}^{N}\left[t_{i} \log Y_{i}+\left(1-t_{i}\right) \log \left(1-Y_{i}\right)\right]-\frac{1}{2} \mathbf{w}^{T} \mathbf{A} \mathbf{w}
\end{aligned}
$$

where, $\mathbf{A}=\operatorname{diag}\left(\alpha_{0}, \alpha_{1}, \cdots, \alpha_{N}\right), Y_{i}=\sigma\left[y\left(\mathbf{x}_{i}, \mathbf{w}\right)\right]$. And $\mathbf{w}_{M P}$ is solved by the second-order Newton method, the gradient vector of $\mathbf{w}$ can be represented as equation (6). Similarly, the Hessian matrix and in formula (5) is given by equation (7) and (8):

$$
\begin{gathered}
\mathbf{g}=\nabla_{w} \log [P(\mathbf{t} \mid \mathbf{w}) p(\mathbf{w} \mid \boldsymbol{\alpha})]=\boldsymbol{\Phi}^{T}(\mathbf{t}-\mathbf{Y})-\mathbf{A} \mathbf{w} \\
\mathbf{H}=\nabla_{w} \nabla_{w} \log \left[P(\mathbf{t} \mid \mathbf{w}) p(\mathbf{w} \mid \boldsymbol{\alpha})=-\boldsymbol{\Phi}^{T} \mathbf{B} \boldsymbol{\Phi}-\mathbf{A}\right. \\
\left\{\begin{array}{l}
\Delta \mathbf{w}=-\mathbf{H}^{-1} \mathbf{g} \\
\mathbf{w}_{M P_{\text {new }}}=\mathbf{w}_{M P}+\Delta \mathbf{w}
\end{array}\right.
\end{gathered}
$$

where, $\quad \mathbf{Y}=\left[Y_{1}, Y_{2}, \cdots, Y_{N}\right]^{T} ; \quad$ and $\mathbf{B}=\operatorname{diag}\left(\beta_{1}, \beta_{2}, \cdots, \beta_{N}\right)$, $\beta_{i}=\left[Y_{i}\left(1-Y_{i}\right)\right] ; \boldsymbol{\Phi}$ is the design matrix, with $\mathbf{\Phi}=\left[\phi\left(x_{1}\right), \phi\left(x_{2}\right), \cdots, \phi\left(x_{N}\right)\right]^{T}$.

2) The posterior probability of Gaussian approximate weight is given by $p(\mathbf{w} \mid \mathbf{t}, \alpha) \approx N\left(\mathbf{w}_{M P}, \Sigma\right)$, where $\mathbf{w}_{M P}$ is posterior probability mean value of weight, and $\sum=\left(-\mathbf{H} \mid \mathbf{w}_{M P}\right)^{-1}$ is a covariance matrix.

3) Using formula (9) to update the hyper parameter $\alpha$ :

$$
\left\{\begin{array}{l}
\alpha_{i}^{n e w}=\frac{\gamma_{i}}{w_{M P i}^{2}} \\
\gamma_{i}=1-\alpha_{i} \Sigma_{i, i}
\end{array}\right.
$$

Where the $\sum_{i, i}$ is the $i^{t h}$ diagonal element, and $w_{M P i}$ is the $i^{\text {th }}$ element for weight vector $\mathbf{w}_{M P}$.

When the hyper parameter $\alpha^{\text {new }}$ is updated, we need re-estimate the posterior mean of weight and calculate the covariance matrix. Repeat steps 1) to 3) until the convergence condition is satisfied. After the update completes, the value of most $\alpha_{i}$ will be close to infinity, the corresponding value of $w_{i}$ will be 0 . As for the remaining $\alpha_{i}$ will take a limited value, and the collection of the corresponding $\mathbf{x}_{i}$ is called Relevance Vector (RV).

\subsection{RVM multi-classification model}

Single relevance vector machine is mainly used for binary classification problem. In this paper, the transformer has been divided into five states. So we built four RVM classifiers based on binary tree architecture to identify the five types:normal state (NS), middle and low thermal overheating (LT), high thermal overheating (HT), high-energy discharge (HD) and low-energy discharge (LD). The RVM1 classifier is trained to distinguish between normal state and fault state. When the input sample is in normal state, output " 0 "; otherwise, output "1". The RVM2 classifier is used to separate the overheat fault from the discharge fault. When input a overheating 
sample to RVM2, output " 0 ", otherwise, output " 1 ". The RVM3 classifier is used to separate middle and low thermal overheating from high thermal overheating. When input a middle and low thermal overheating sample to RVM3, output " 0 "; otherwise, output "1". The RVM4 classifier is used to separate low-energy discharge from high-energy discharge. When input a low-energy discharge sample to RVM4, output " 0 "; otherwise, output "1". The flowchart of multi-layer classification model based on RVM is shown in Figure 1.

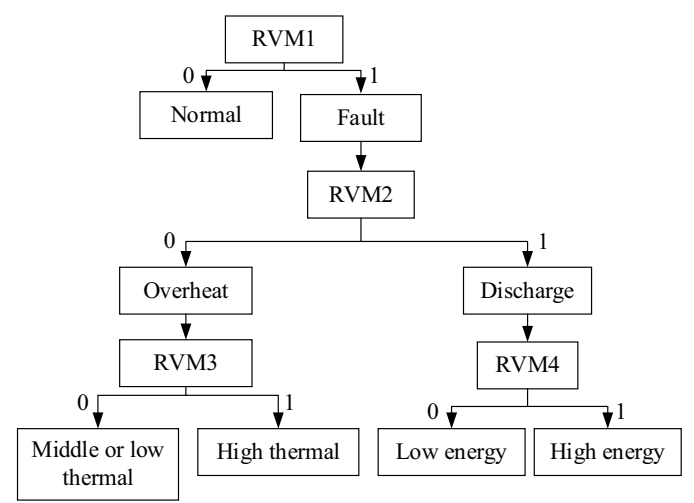

Figure 1. Transformer fault diagnosis model based on RVM.

\section{Transformer fault diagnosis based on CRO-RVM model}

\subsection{Fault feature vector extraction}

In this paper, we chose 5 diagnostic gases as input vector of RVM classifier. These gases include hydrogen $\left(\mathrm{H}_{2}\right)$, methane $\left(\mathrm{CH}_{4}\right)$, ethane $\left(\mathrm{C}_{2} \mathrm{H}_{6}\right)$, ethylene $\left(\mathrm{C}_{2} \mathrm{H}_{4}\right)$ and acetylene $\left(\mathrm{C}_{2} \mathrm{H}_{2}\right)$. In order to reduce the calculation error caused by the difference of the DGA data, the training set data and testing-set data should be standardized, it is shown as the equation (10):

$$
x_{i j}^{*}=\frac{x_{i j}}{x_{j}}
$$

Where, $x_{i j}$ is the volume fraction of the $j^{\text {th }}$ gas in the $i^{\text {th }}$ sample, and $j \in\{1,2, \cdots, 5\} . x_{j}$ is alarm value of the $j^{\text {th }}$ gas refer to $G B / T$ 7252-2001 Guide to the Analysis and the Diagnosis of Gases Dissolved in Transformer Oil, and $x_{i j}^{*}$ is called gas deterioration degree.

\subsection{Chemical reaction optimization algorithm (CRO)}

$\mathrm{CRO}$ is a meta-heuristic algorithm which has been developed in recent years. It has been put forward by Albert Y.S.Lam and Victor O.K.Li from the University of Hong Kong [12]. It simulates molecular motion and energy conversion processes in chemical reactions. Compared with genetic algorithm and particle swarm optimization algorithm $[13,14]$, it is characterized by not only the dynamic change of group size, but also the rich information interactive modes among individuals.

There are two basic arithmetic units in CRO algorithm, one is called molecule $(\omega)$ and the other is named container wall (buffer). Molecules have two kinds of energy are called kinetic energy (KE) and potential energy (PE). Buffer represents the reaction environment, $\mathrm{PE}$ is the measure index in CRO algorithm. When CRO algorithm is applied to solve a real problem, PE represents the objective function. $\mathrm{KE}$ is the quantitative value to judge whether the molecular reaction can be carried out. There are four basic reaction operators in CRO: single-molecule collision, single-molecule decomposition, inter-molecular collision and molecular synthesis $[15,16]$.

The chemical reaction algorithm first used the strength strategy formed by invalid collision to search the adjacent region. When a lower energy state cannot be found in the neighborhood, it will use the diversification strategy formed by inter-molecular collision and molecular synthesis to jump to a relatively remote areas continue to search. In the search process, the energy will be redistributed among molecules in various ways.

The choice of the initial value of the algorithm has a great influence on the results. In order to make the algorithm have better convergence speed, search precision and optimization ability, the initial molecular population is generated in a random way. The related parameters include molecular population size (Popsize), central energy buffer (Buffer), molecular initial kinetic energy (initialKE), molecular kinetic energy loss rate (KelossRate), etc. The specific steps of parameter setting are as follows:

1) Form a random molecule in a random manner within the range of molecular properties;

2) Judge whether the random molecule to meet the constraints or not, if satisfied, then enter step 3), otherwise, return to step 1);

3) Calculate the molecular potential energy, set the initial kinetic energy of the molecule, and save the molecule into the reaction container. At the same time, verify whether the initial molecular population in the container to achieve the initial set size or not, if reached, then finish the initialization process, otherwise, return to step 1).

In this paper, the main parameters of $\mathrm{CRO}$ algorithm are assigned as follows: Popsize $=10$; initialKE $=1500$; Kelossrate $=0.25$ and Buffer $=0$.

\subsection{The diagnosis steps of transformer fault based on CRO-RVM model}

Transformer fault diagnosis based on CRO-RVM model used CRO algorithm to optimize the kernel function parameters of the RVM classifiers. The flowchart is shown in Figure 2, the specific steps are described as follows:

1) The sample data are selected and normalized to obtain the feature vector. We divided the sample data into training set and test set according to the ratio of 2: 1 . 
2) Use the binary tree method shown in Figure 1 to establish a multi-level transformer fault diagnosis model based on RVM, and a set of kernel function parameters are initialized, then inputting the training set to train the model.

3) Initialize the chemical reaction optimization algorithm, select the mean square error of the RVM's actual output and expected output as the objective function.

4) Calculate the initial potential energy and initial kinetic energy of the molecule. Optimize the kernel function parameters by iterative calculation.

5) When the PE of molecule is smallest, parameters will obtain the global optimal solution. Assign these kernel function parameters to corresponding RVM classifiers.

6) Input the test data sets into the CRO-RVM model, and test its diagnostic accuracy.

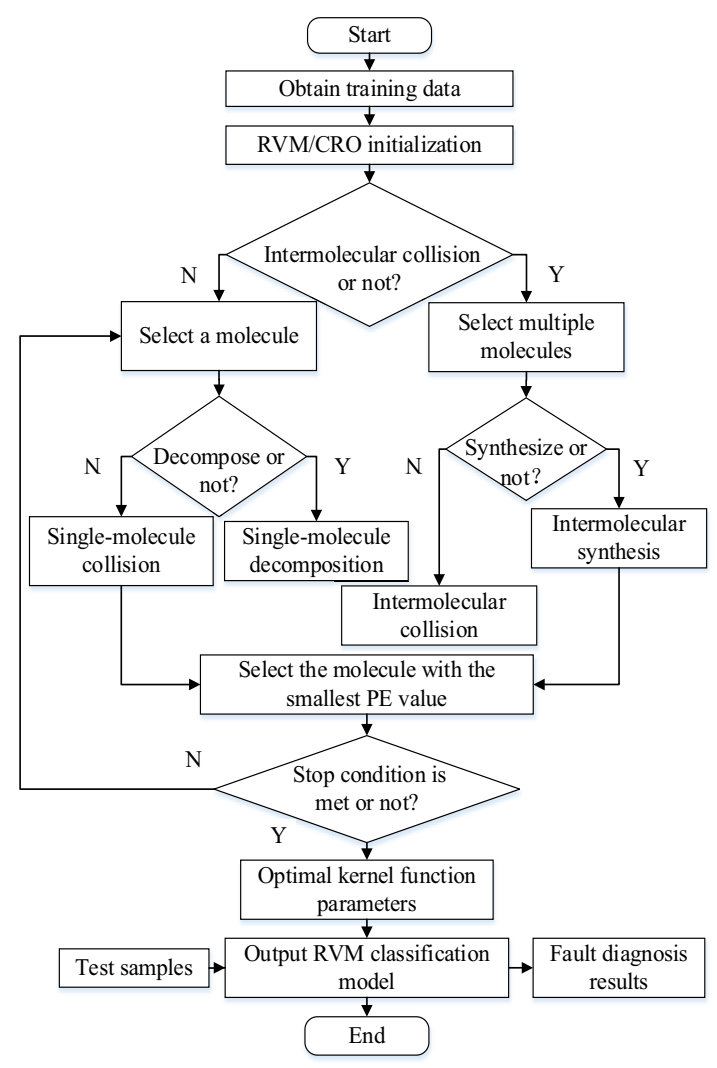

Figure 2. Diagnosis flowchart of CRO-RVM model.

\section{Comparison of different diagnostic models and example analysis}

\subsection{Comparison of different diagnostic models}

In this paper, we collected 300 sets of measured DGA data from the $220 \mathrm{kV}, 330 \mathrm{kV}$ and $500 \mathrm{kV}$ transformers. The 15 sets of measured data samples shown in Table 1 are part of them. We divided all of them into training set and testing set according to the ratio of $2: 1$. We used IEC three-ratio method, RVM model and CRO-RVM model to train and diagnose these data samples. The kernel functions of RVM model and CRO-RVM model both are
$\mathrm{RBF}$, and the kernel function parameters of the RVM model all are 0.5 . The four classifiers' parameters of the CRO-RVM model are optimized by CRO algorithm, they are $[8.625,0.264,0.516,1.246]$. Figure 3 shows the mean square error performance of the CRO-RVM model is changed with the iteration number. The results of the three diagnostic methods are shown in Table 2 as follows.

Table 1. DGA data samples of power transformer

\begin{tabular}{|c|c|c|c|c|c|}
\hline \multirow{2}{*}{$\begin{array}{c}\text { Transformer } \\
\text { state }\end{array}$} & \multicolumn{5}{|c|}{ Diagnosis gas content $(\mu \mathrm{L} / \mathrm{L})$} \\
\cline { 2 - 6 } & $\mathrm{H}_{2}$ & $\mathrm{CH}_{4}$ & $\mathrm{C}_{2} \mathrm{H}_{6}$ & $\mathrm{C}_{2} \mathrm{H}_{4}$ & $\mathrm{C}_{2} \mathrm{H}_{2}$ \\
\hline $\mathrm{NS}$ & 13.6 & 5.3 & 8.2 & 29 & 2.1 \\
\hline $\mathrm{NS}$ & 32 & 11.38 & 8.2 & 30.6 & 3.9 \\
\hline $\mathrm{NS}$ & 5.8 & 2.7 & 1.8 & 0.8 & 0 \\
\hline LT & 13.1 & 44.7 & 91.5 & 213.3 & 4.2 \\
\hline LT & 70.2 & 246.6 & 171.5 & 371.6 & 0 \\
\hline LT & 93 & 58 & 43 & 37 & 0 \\
\hline HT & 39.4 & 40.6 & 17.2 & 51.4 & 4.3 \\
\hline HT & 8.4 & 28.7 & 13 & 105 & 2.1 \\
\hline HT & 170 & 320 & 53 & 520 & 3.2 \\
\hline HD & 263 & 628 & 16.8 & 46 & 855.4 \\
\hline HD & 354 & 387 & 65.8 & 101.2 & 189 \\
\hline HD & 158 & 71 & 1.3 & 146 & 685 \\
\hline LD & 97.8 & 15.9 & 2.7 & 8.1 & 24.3 \\
\hline LD & 66 & 8.3 & 8.2 & 9.2 & 8.2 \\
\hline LD & 59 & 10.4 & 4 & 10 & 12.7 \\
\hline
\end{tabular}

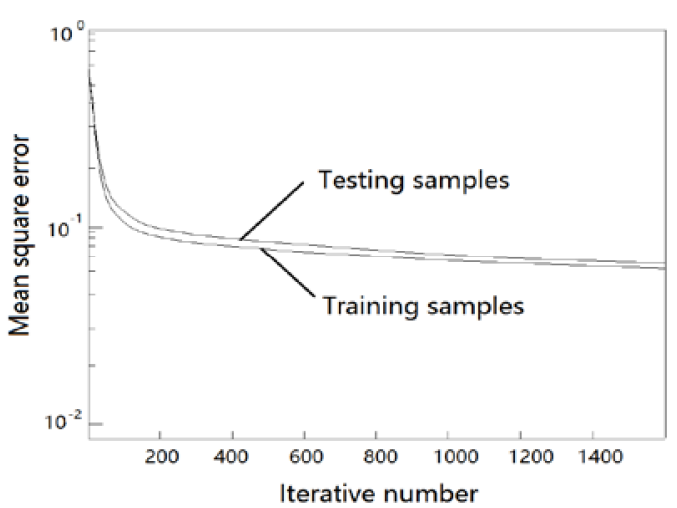

Figure 3. Mean square error variation trend of CRO-RVM model.

Table 2. Comparison of fault diagnosis results.

\begin{tabular}{|c|c|c|c|c|}
\hline state & $\begin{array}{c}\text { Sample } \\
\text { Size }\end{array}$ & IEC/\% & RVM/\% & CRO-RVM/\% \\
\hline NS & 10 & 60 & 100 & 100 \\
\hline LT & 16 & 100 & 81.3 & 93.8 \\
\hline HT & 20 & 80 & 80 & 90 \\
\hline HD & 40 & 37.5 & 72.5 & 87.5 \\
\hline LD & 14 & 42.9 & 57.1 & 100 \\
\hline Total & 100 & $64.08^{*}$ & 78.18 & 94.26 \\
\hline
\end{tabular}

(The symbol * indicates the presence of unrecognized samples.)

We can get the following conclusions from the diagnostic results based on different models:

1) The IEC three-ratio method has the problem of missing codes. For example, [354, 387, 65.8, 101.2, 189] is a group of high-energy discharge data. According to the coding rules shown in table 3 , it is obviously out of the coding range. So the IEC three-ratio method can't diagnose it. The RVM model and the CRO-RVM model 
solve this problem perfectly, they can identify this kind of data sample correctly so as to decrease the misdiagnosis rate.

Table 3. Coding rules of the IEC three-ratio method

\begin{tabular}{|c|c|c|c|}
\hline Fault types & $\mathrm{C}_{2} \mathrm{H}_{2} / \mathrm{C}_{2} \mathrm{H}_{4}$ & $\mathrm{CH}_{4} / \mathrm{H}_{2}$ & $\mathrm{C}_{2} \mathrm{H}_{2} / \mathrm{C}_{2} \mathrm{H}_{6}$ \\
\hline LT & $<0.1$ & $>1$ & $1-4$ \\
\hline $\mathrm{HT}$ & $<0.2$ & $>1$ & $>4$ \\
\hline $\mathrm{HD}$ & $0.6-2.5$ & $0.1-1$ & $>2$ \\
\hline LD & $>1$ & $0.1-0.5$ & $>1$ \\
\hline $\begin{array}{c}\text { unrecognized } \\
\text { sample }\end{array}$ & 1.53 & 1.09 & 2.87 \\
\hline
\end{tabular}

2) The kernel function parameters in RVM model are fixed, so it is difficult to ensure the classifiers in different levels can achieve the best classification results at the same time. So the diagnostic accuracy of the RVM model is only about $78 \%$.

3) After using CRO algorithm to optimize the kernel function parameters of the RVM classification model, the diagnostic accuracy has been obviously improved, reached more than $94 \%$. The correct recognition rate meets the requirement of practical application.

\subsection{Transformer fault diagnosis case}

The No.1 main transformer in a $330 \mathrm{kV}$ substation was broken down in June 25, 2014. With the transformer heavy gas operation, its three side switch tripped. The main transformer was made by Xi'an XD Transformer Co., Ltd, its model number is OSFPSZ9-M-360000/330. It has been put into operation since 2006. Oil chromatographic test data is shown in Table 4.

Table 4. DGA test data

\begin{tabular}{|c|c|c|c|c|c|c|}
\hline \multirow{2}{*}{$\begin{array}{c}\text { Test } \\
\text { time }\end{array}$} & \multicolumn{5}{|c|}{ Gas content $(\mu \mathrm{L} / \mathrm{L})$} & \multirow{2}{*}{ Remark } \\
\cline { 2 - 6 } & $\mathrm{H}_{2}$ & $\mathrm{CH}_{4}$ & $\begin{array}{c}\mathrm{C}_{2} \mathrm{H} \\
6\end{array}$ & $\begin{array}{c}\mathrm{C}_{2} \mathrm{H} \\
4\end{array}$ & $\begin{array}{c}\mathrm{C}_{2} \mathrm{H} \\
2\end{array}$ & \\
\hline $2014-5$ & 8.0 & 2.6 & 0.6 & 1.0 & 0 & Pre-fault \\
\hline $2014-6$ & 2142 & 234 & 4.5 & 57.4 & 86.9 & $\begin{array}{c}\text { After- } \\
\text { fault }\end{array}$ \\
\hline
\end{tabular}

The CRO-RVM model proposed in this paper is applied to analyze these DGA test data. The output diagnosis result is $\mathrm{HD}$, that means the fault type of No.1 transformer is high-energy discharge. After disassembling inspection, we found there were discharge marks at the bottom of transformer bushing and the end of current-carrying rod. And there was insulation breakdown in B phase winding. So the real fault type of this transformer is high-energy discharge. It perfectly confirms the prediction of the CRO-RVM model. The disassembling picture of No.1 transformer is shown in Figure 4.
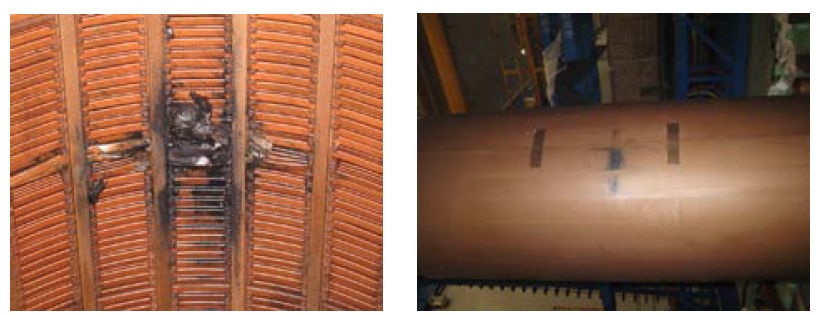

Figure 4. Disassembling picture of transformer 1.

The analysis of above case indicates that the transformer fault diagnosis method we proposed not only has good theoretical feasibility, but also has splendid ability of practical application. The new method can be applied to identify multiple fault types of large power transformer.

\section{Conclusions}

Relevance Vector Machine (RVM) is one of the emerging algorithms in machine learning field. The use of RVM in transformer fault diagnosis has been paid more and more attention. Based on the standard RVM classification model, this paper proposed a CRO-RVM model for transformer fault diagnosis. Using the CRO algorithm to optimize RVM kernel function parameters and improve the classification correct rate. Through the comparative analysis of the diagnosis results by different methods, we found that the CRO-RVM model can fully consider the individual differences of different transformers, and makes a relatively accurate diagnosis. Compared with the IEC three-ratio method, it doesn't has the problem of missing codes. And its diagnostic accuracy is higher than the standard RVM model. And a fault diagnosis case shows that the new method we presented is suitable for practical application. Its predicted results accord well with the real situation. The research indicates that it is a more useful tool for transformer fault diagnosis. In the future, we will try to combine DGA data with the routine electrical test data as fault features to further improve the diagnostic performance of the CRO-RVM model. In addition, we will study how to apply it to diagnose the fault of other oil-filled electrical equipment.

\section{References}

1. C.X. Guo, Y. Wang, M. Wang, “Time-varying Outage Model for Transformers Representing Internal Latent fault," Proc. Chin. Soc. Electrical. Eng. 33, 1 (2013)

2. F. Jakob, J.J. Dukarm, "Thermodynamic Estimation of Transformer Fault Severity," IEEE Trans. Power. Del. 30, 4, 1941-1948 (2015)

3. S. Bhowmick, S. Nandi, "Online Detection of an Interturn Winding Fault in Single-Phase Distribution Transformers Using a Terminal Measurement-Based Modeling Technique," IEEE Trans. Power. Del, 30, 2, 1-9 (2015)

4. Z.X. Liu, B. Song, E.W. Li, et al. "Study of "code absence" in the IEC three-ratio method of dissolved gas analysis," IEEE Electron. Insulat. Maga. 31, 6, 6$12(2015)$

5. P.V.B. Reddy, C.R.V. Kumar, K.H. Reddy, "Modeling of Surface Roughness in Wire Electrical Discharge Machining Using Artificial Neural Networks," Int. J. Mech. Eng. \&Rob. Res. 2, 1, 5764 (2013)

6. K. Bacha, S. Souahlia, M. Gossa, "Power transformer fault diagnosis based on dissolved gas 
analysis by support vector machine,". Electron. Power. Sys. Res. 83, 1, 73-79 (2012)

7. A.M. Shah, B.R. Bhalja, "Discrimination Between Internal Faults and Other Disturbances in Transformer Using the Support Vector MachineBased Protection Scheme," IEEE Trans. Power. Del. 28, 3, 1508-1515 (2013)

8. S. Hwang,M.K. Jeong, B.J. Yum, "Robust Relevance Vector Machine With Variational Inference for Improving Virtual Metrology Accuracy," IEEE Trans. Semiconduct. Manu. 27, 1, 83-94 (2014)

9. M.E. Tipping, "Sparse bayesian learning and the relevance vector machine," J. Mach. Learn. 1, 3, 211244 (2001)

10. J. Yin, Y. Zhu, Y.U. Guoqin. "Relevance vector machine and its application in transformer fault diagnosis," Power. Sys. Techno. 32, 12, 2239-2247 (2012)

11. T. Wang,H. Xu,J. Han, et al. "Cascaded H-Bridge Multilevel Inverter System Fault Diagnosis Using a PCA and Multi-class Relevance Vector Machine Approach," IEEE Trans. Power. Electron. 30, 12, (2015)
12. A.Y.S Lam, V.O.K Li, "Chemical-reaction-inspired metaheuristic for optimization," IEEE Trans. Evol. Comput. 14, 3, 381-399 (2010)

13. P. Sreeraj, T. Kannan, S. Maji, "Simulation and Parameter Optimization of GMAW Process Using Neural Networks and Particle Swarm Optimization Algorithm," Int. J. Mech. Eng. \&Rob. Res. 2, 1, 130146 (2013)

14. M. Adinarayana, G. Prasanthi, G. Krishnaiah, "Optimization for Surface Roughness, MRR, Power Consumption in Turning of En24 Alloy Steel Using Genetic Algorithm," Int. J. Mech. Eng. \&Rob. Res. 3, 1, 20-26 (2014)

15. H.L. Ao, J. Cheng, J. Zheng, et al. "Roller Bearing Fault Diagnosis Method Based on Chemical Reaction Optimization and Support Vector Machine," J. Comput. Civil. Eng. 29, 5, (2014)

16. H. Duan, L. Gan, "Orthogonal Multiobjective Chemical Reaction Optimization Approach for the Brushless DC Motor Design," IEEE T. Magn. 51, 1, 1-7 (2015) 